\title{
A Personal Sound Amplification Product Compared to a Basic Hearing Aid for Speech Intelligibility in Adults with Mild-to-Moderate Sensorineural Hearing Loss
}

\author{
Ji Eun Choi ${ }^{1}$, Jinryoul Kim², Sung Hoon Yoon ${ }^{2}$, Sung Hwa Hong ${ }^{3}$, and II Joon Moon ${ }^{4}$ \\ ${ }^{1}$ Department of Otorhinolaryngology-Head and Neck Surgery, Dankook University Hospital, Cheonan, Korea \\ ${ }^{2}$ Hearing Research Laboratory, Samsung Medical Center, Seoul, Korea \\ ${ }^{3}$ Department of Otorhinolaryngology-Head \& Neck Surgery, Samsung Changwon Hospital, School of Medicine, \\ Sungkyunkwan University, Changwon, Korea \\ ${ }^{4}$ Department of Otorhinolaryngology-Head and Neck Surgery, Samsung Medical Center, School of Medicine, Sungkyunkwan University, \\ Seoul, Korea
}

Received September 2, 2019

Revised October 10, 2019

Accepted October 21, 2019
Background and Objectives: This study aimed to compare functional hearing with the use of a personal sound amplification product (PSAP) or a basic hearing aid (HA) among sensorineural hearing impaired listeners. Subjects and Methods: Nineteen participants with mild-to-moderate sensorineural hearing loss (SNHL) (26-55 dB HL; pure-tone average, $0.5-4 \mathrm{kHz})$ were prospectively included. No participants had prior experience with HAs or PSAPs. Audiograms, speech intelligibility in both quiet and noisy environments, speech quality, and preference were assessed in three different listening conditions: unaided, with the HA, and with the PSAP. Results: The use of PSAP was associated with significant improvement in pure-tone thresholds at 1, 2, and $4 \mathrm{kHz}$ compared to the unaided condition (all $p<0.01$ ). In the quiet environment, speech intelligibility was significantly improved after wearing a PSAP compared to the unaided condition $(p<0.001)$, and this improvement was better than the result obtained with the HA. The PSAP also demonstrated similar improvement in the most comfortable levels compared to those obtained with the HA $(p<0.05)$. However, there was no significant improvement of speech intelligibility in a noisy environment when wearing the PSAP $(p=0.160)$. There was no significant difference in the reported speech quality produced by either device or in participant preference for the PSAP or HA. Conclusions: The current result suggests that PSAPs provide considerable benefits to speech intelligibility in a quiet environment and can be a good alternative to compensate for mild-to-moderate SNHL.

J Audiol Otol 2020;24(2):91-98 Mild-to-moderate sensorineural hearing loss.

\section{Introduction}

Hearing impairment is known to limit communication and social activity [1], leading to a lower quality of life and decreased cognitive function [2]. In the past, the majority of surveys on the need for hearing rehabilitation covered only bilateral hearing loss greater than $40 \mathrm{~dB}$. However, recent

This is an Open Access article distributed under the terms of the Creative Commons Attribution Non-Commercial License (https://creativecommons.org/licenses/by-nc/4.0/) which permits unrestricted non-commercial use, distribution, and reproduction in any medium, provided the original work is properly cited. studies have reported that individuals with minimal to mild hearing loss also have difficulties understanding speech under adverse listening conditions and have a poorer quality of life $[3,4]$. Hearing aids (HAs) are an effective rehabilitative option for improving communication as well as the psychosocial well-being of individuals with hearing loss. While the benefits of HAs have been well-documented, less than $20 \%$ of adults with hearing loss was reported as HA users $[5,6]$. Furthermore, only $0.47 \%$ of individuals with minimal hearing loss reported using HAs even when they suffered subjective hearing difficulty [7]. Cost is considered to be one of the rea- 
sons for the low adoption rate of HAs [8]. According to the MarkeTrak VII survey, $76 \%$ of non-adapters mentioned finances as a barrier to the adoption of conventional HAs [9].

Personal sound amplification products (PSAPs) are defined by the US Food and Drug Administration (FDA) as wearable electronic products intended to be used by consumers without hearing impairment to amplify sounds in certain environments [10]. This specific category of amplification is separate from traditional HAs, as PSAPs are not meant to be used to compensate for hearing loss and are therefore not regulated as a medical device by the US FDA [11]. Because PSAPs are intended to be sold directly to consumers, they can be considered a type of over-the-counter (OTC) amplification device. In general, PSAPs are less expensive and simpler sound amplification devices with fewer features and less functionality than digital HAs. However, some PSAPs are technologically comparable with basic HAs. Although PSAPs are not intended to compensate for impaired hearing, it was found that approximately 1.5 million people with hearing impairment use either a PSAP or OTC device to compensate for their hearing loss [12].

Previous research suggested that certain higher-end PSAPs may be appropriate to use for those with mild-to-moderate hearing loss [12-14]. A more recent study has compared five PSAPs with a conventional HA in older adults with mild-tomoderate hearing loss [15]. Results in speech recognition performance in noise indicated that three of the five PSAPs performed comparable to an HA. However, many studies focused on electroacoustic characteristics. There is still insufficient data on PSAP performance. Additionally, other studies showed that PSAPs do not provide sufficient gain for high frequencies, so people have very low satisfaction with PSAPs $[16,17]$. Therefore, a conventional HA and a PSAP need to be compared using a comprehensive method. The present study evaluated the performance of a PSAP and a basic HA among adults with mild-to-moderate hearing loss.

\section{Subjects and Methods}

\section{Participants}

From October 2015 through January 2016, a total of 19 participants with no experience with any hearing assistive devices, such as HAs or PSAPs, were prospectively enrolled. Patients with otitis media and conductive hearing loss were excluded from the study. All participants had mild-to-moderate sensorineural hearing loss (SNHL) (26-55 dB HL; pure-tone average from $500-4,000 \mathrm{~Hz}$ ) in the tested ear. If the participants had mild-to-moderate SNHL in both ears, the ear with better hearing was selected as the tested ear.

\section{Ethical statement}

All participants were recruited and tested at the Samsung Medical Center Hearing Research Laboratory. Every participant provided written informed consent to participate in this study. The study protocol was approved by the Samsung Medical Center Institutional Review Board (IRB No. 2015-06-064). This study was carried out in accordance with approved guidelines.

\section{Intervention}

Participants were fitted with a PSAP (PS2500AMP, Able Planet, North Wheat Ridge, CO, USA) and an HA (Audéo ${ }^{\mathrm{TM}}$ Q, Phonak AG, Stäfa, Switzerland) unilaterally. The Able Planet PS2500AMP (Able Planet) in-ear amplifier has 8 channels with 12 gain adjustment bands, noise reduction, feedback cancellation, and directionality. The participants selected their preferred amplification mode among 4 presets after listening to the Korean standard sentence lists for adults (KS-SL-A) of the Korean Standard Audiometry (KSA) test [18] in a quiet environment. The PSAP contains a 'natural directionality' feature as the default, and its settings could not be modified. The Phonak Audéo ${ }^{\mathrm{TM}} \mathrm{Q}$ (Phonak AG) HA was also a canal-style receiver with 8 channels. The HA was fitted to the National Acoustic Laboratories' non-linear prescription, version 2 (NALNL2) formula by an experienced audiologist [19,20]. All functions of the HA were disabled apart from noise reduction, automatic directionality, and feedback cancellation.

The Frye Fonix ${ }^{\circledR} 7000$ Hearing Aid Test system (Frye Electronics, Beaverton, OR, USA) was used for measuring coupler gain. Multi-curve coupler gain of digital speech was analyzed at input levels of 40, 60, and $80 \mathrm{~dB}$ sound pressure level (SPL). The coupler gain of the HA was measured for each participant in accordance with NAL-NL2, and the coupler gain of the PSAP was measured for each amplification mode. Participants were blinded to information about the hearing devices.

\section{Outcome measures}

Four main test batteries were administered: 1) an audiogram, 2) speech intelligibility in a quiet environment, 3) speech intelligibility in a noisy environment, and 4) a self-rating questionnaire. Participant performance was compared across three different listening conditions: unaided, with an HA, and with a PSAP. All tests were administered monoaurally, and a ear plug was inserted in the non-tested ear. The order of wearing devices was randomized.

\section{Audiograms}

Pure-tone threshold and word recognition score (WRS) were measured using a Madsen Orbiter 922 audiometer (Madsen Electronics, Taastrup, Denmark) and TDH-39P headphones 
(Telephonics, Farmingdale, NY). Pure-tone thresholds were obtained at $0.25,0.5,1,2,4$, and $8 \mathrm{kHz}$ using an ascending 5-dB method (modified Hughson-Westlake method) [21]. The WRS was measured using KS-MWL-A [22] at the most comfortable level (MCL).

\section{Speech intelligibility}

Speech reception thresholds (SRTs) were measured in quiet and noisy test conditions using the Korean version of Hearing in Noise Test (K-HINT) sentences [22]. The study participant sat on a chair in the center of the sound field facing 2 loudspeakers (HS-50M, Yamaha, Japan) located approximately $1 \mathrm{~m}$ away at the $0^{\circ}$ azimuth. The target sentences in K-HINT and speech-shaped noise were presented from two front loudspeakers $\left(0^{\circ}\right)$. The presentation level of the noise was fixed at $65 \mathrm{dBA}$, and the level of target sentences varied according to the adaptive procedure of the original HINT [22]. One list of twenty sentences was randomly selected from a total of twelve lists. Results of the K-HINT were presented as dB HL and SNR; speech levels corresponding to $50 \%$ correct recognition of sentences in quiet and noisy testing condition, respectively.

\section{Speech quality}

Speech quality was assessed using the eighth KS-SL-A of the KSA test and speech-shaped noise (0-dB SNR). Participants were asked to rate the quality of sound using a five-point numerical scale recommended by the International Telecommunication Union (ITU) $[23,24]$. The quality of speech was assessed by rating speech signal alone, the background noise alone, and the overall effect of speech and noise on quality using the following parameters: 1) the speech signal alone using an absolute category rating (ACR, "Excellent," "Good," "Fair," "Poor," and "Bad"), 2) the speech signal alone using a scale of signal distortion (SIG, "Not distorted," "Slightly distorted," "Somewhat distorted," "Fairly distorted," and "Very distorted"), 3) the background noise alone using a scale of background intrusiveness (BAK, "Not noticeable," "Slightly noticeable," "Noticeable but not intrusive," "Somewhat intrusive," and "Very intrusive"), and 4) the overall effect of speech and noise on quality (OVL, "Excellent," "Good," "Fair," "Poor," and "Bad"). The five-level categorical scales were converted to numerical scores, where "Excellent," "Not distorted," or "Not noticeable" corresponded to a score of 5 and "Bad," "Very distorted," or "Very intrusive" to 1 . The mean opinion score (MOS) is obtained from the four component scores (ACR, SIG, BAK, and OVL) was determined as follows: $\operatorname{MOS}=\Sigma^{N}{ }_{n=1}$ $R_{n} / N$, where $R_{n}$ represents the individual ratings for a given stimulus by $N$ subjects.

\section{Preference test}

Device preference was assessed using a comparison category rating (CCR) under quiet and noisy test conditions (speechshaped noise, $0 \mathrm{~dB}$ SNR). Participants were asked to listen to the first and second KS-SL-A test at the MCL, and to select their preferred device (HA or PSAP). The CCR test is designed to quantify the degree of preference on a six-point scale with the rating of 0 indicating "No device preference," 1 indicating "Very slightly better," 2 indicating "Slightly better," 3 indicating "Better," 4 indicating "Much better," and 5 indicating "Very much better." Positive and negative numbers are used to account for both directions of preference (positive for HA and negative for PSAP). The resulting preference value of the CCR test is presented as a comparison mean opinion score (CMOS).

\section{Statistical analysis}

Results were analyzed using SPSS 18.0 (SPSS Inc., Chicago, IL, USA). To compare the pure-tone thresholds, WRS, MCL, speech intelligibility, and speech quality among the three listening conditions (unaided, with the HA, and with the PSAP), repeated measures one-way analysis of variance (RM-ANOVA) or Friedman tests were conducted depending on normality. If there were significant differences among the three listening conditions, post hoc tests using the Bonferroni correction or Wilcoxon signed-rank test were performed to evaluate differences between two different listening conditions (i.e., unaided vs. HA-aided, unaided vs. PSAP-aided, and HA-aided vs. PSAP-aided). Post hoc analyses with Wilcoxon signedrank tests were conducted with the Bonferroni correction applied, resulting in a significance level of $p<0.017$.

\section{Result}

\section{Participants}

A total of 19 participants (4 males, 15 females, age range 35-86 years, average 64 years) with mild-to-moderate SNHL were prospectively enrolled. Individual clinical and device information is presented in Table 1. The preferred amplification mode for the PSAP was Program $1(n=10)$, followed by Program $3(n=5)$, Program $4(n=3)$, and Program $2(n=1)$.

\section{Coupler gain}

Fig. 1A-D shows coupler gain for the PSAP according to amplification mode at inputs of 40,60 , or $80 \mathrm{~dB}$ SPL. Fig. $1 \mathrm{E}$ shows mean coupler gains with $95 \%$ confidence intervals for the HA at inputs of 40,60 , or $80 \mathrm{~dB}$ SPL after fitting with NALNL2. Most gain programs in PSAP prescribed similar coupler gains throughout all frequencies, except at $8 \mathrm{kHz}$. On the other hand, the HA fitted with NAL-NL2 provided greater cou- 
Table 1. Demographics and device information

\begin{tabular}{|c|c|c|c|c|c|c|c|c|}
\hline \multirow[b]{2}{*}{ Participants } & \multirow[b]{2}{*}{ Sex } & \multirow{2}{*}{$\begin{array}{c}\text { Age } \\
\text { (years) }\end{array}$} & \multirow[b]{2}{*}{ Tested ear } & \multicolumn{2}{|c|}{ PTA_4FA } & \multicolumn{2}{|c|}{$\mathrm{DHA}$} & \multirow{2}{*}{$\begin{array}{c}\text { PSAP } \\
\text { Selected } \\
\text { program }\end{array}$} \\
\hline & & & & Right & Left & Receiver type & Dome type & \\
\hline S1 & $\mathrm{F}$ & 54 & L & 31.3 & 27.5 & Standard & Open & 1 \\
\hline S2 & $\mathrm{F}$ & 35 & $R$ & 28.8 & 35.0 & Standard & Close & 1 \\
\hline S3 & $\mathrm{F}$ & 74 & $R$ & 37.5 & 42.5 & Standard & Close & 1 \\
\hline S4 & $\mathrm{F}$ & 51 & L & 25.0 & 28.8 & Standard & Close & 1 \\
\hline S5 & $\mathrm{F}$ & 86 & $R$ & 43.8 & 48.8 & Standard & Power & 2 \\
\hline S6 & $M$ & 59 & $R$ & 35.0 & 40.0 & Standard & Open & 1 \\
\hline S7 & $\mathrm{F}$ & 67 & L & 41.3 & 35.0 & Standard & Open & 4 \\
\hline S8 & $\mathrm{F}$ & 69 & L & 50.0 & 42.5 & Standard & Power & 3 \\
\hline 59 & $\mathrm{F}$ & 59 & $R$ & 36.3 & 50.0 & Standard & Open & 3 \\
\hline$S 10$ & $M$ & 64 & L & 46.3 & 41.3 & Standard & Open & 1 \\
\hline S11 & M & 67 & $R$ & 43.8 & 45.0 & Standard & Power & 1 \\
\hline$S 12$ & $\mathrm{~F}$ & 59 & L & 36.3 & 30.0 & Standard & Closed & 1 \\
\hline$\$ 13$ & $\mathrm{~F}$ & 73 & $R$ & 31.3 & 30.0 & Standard & Open & 3 \\
\hline S14 & $\mathrm{F}$ & 68 & L & 36.3 & 31.3 & Standard & Open & 1 \\
\hline S15 & $F$ & 70 & $R$ & 33.8 & 42.5 & Standard & Open & 3 \\
\hline$S 16$ & $\mathrm{~F}$ & 67 & L & 25.0 & 28.8 & Standard & Open & 3 \\
\hline S17 & M & 58 & $R$ & 35.0 & 56.3 & Standard & Open & 4 \\
\hline$S 18$ & $F$ & 57 & $R$ & 35.0 & 27.5 & Standard & Open & 4 \\
\hline S19 & $\mathrm{F}$ & 70 & $\mathrm{R}$ & 36.3 & 40.0 & Standard & Closed & 1 \\
\hline
\end{tabular}

PTA: pure-tone audiometry, 4FA: averaged for $0.5 \mathrm{k}, 1 \mathrm{k}, 2 \mathrm{k}$, and $4 \mathrm{k} \mathrm{Hz}$, DHA: digital hearing aid, PSAP: personal sound amplification product, F: female, M: male, R: right, L: left

pler gain above $1 \mathrm{kHz}$ than below $1 \mathrm{kHz}$.

\section{Audiograms}

Pure-tone thresholds (Fig. 2A), MCL (Fig. 2B), and WRS (Fig. 2C) were compared between unaided, HA-aided, and PSAP-aided conditions. There were significant differences in pure-tone thresholds among the three listening conditions at $0.5,1,2,4$, or $8 \mathrm{kHz}$ (all $p<0.01$ in RM-ANOVA). Bonferronicorrected post hoc tests revealed the PSAP significantly improved hearing thresholds at 1,2 , or $4 \mathrm{kHz}$ compared to the unaided condition (all $p<0.01$ ), and it provided significantly greater hearing gain at 2 and $4 \mathrm{kHz}$ than did the HA ( $p=0.045$ and $<0.001$, respectively). However, the PSAP provided no significant hearing gain at $8 \mathrm{kHz}$ compared to the unaided condition ( $p=0.865$ ). The HA provided a significant hearing gain at $8 \mathrm{kHz}$ compared to the unaided $(p<0.01)$ and PSAPaided conditions $(p=0.006)$. Mean thresholds of MCL in unaided, HA-aided, and PSAP-aided conditions were $66 \mathrm{~dB}, 53$ $\mathrm{dB}$, and $55 \mathrm{~dB}$, respectively. There were statistically significant differences in MCL depending on the listening condition $\left[\chi^{2}(2)=33.343, p<0.001\right]$. Both aided conditions significantly reduced the thresholds of MCL compared to the unaided condition $(Z=-3.839, p<0.001$ for HA-aided vs. unaided, $Z=$
$-3.765, p<0.001$ for PSAP-aided vs. unaided). The thresholds of MCL in the HA was significantly lower than those of the $\operatorname{PSAP}(Z=-2.460, p=0.014)$. However, a Friedman test determined that there was no significant difference in WRSs depending on listening conditions $\left[\chi^{2}(2)=1.582, p=0.454\right]$.

\section{Speech intelligibility}

Fig. 3A shows the SRTs obtained in a quiet condition. A repeated measures ANOVA determined that SRTs in a quiet condition differed significantly between listening conditions $[\mathrm{F}(2,36)=19.674, p<0.001]$. Bonferroni-corrected post hoc tests revealed that the HA elicited a slight reduction in SRTs in a quiet environment compared to the unaided condition $(38.5 \pm 5.7 \mathrm{~dB}$ HL vs. $39.9 \pm 8.0 \mathrm{~dB}$ HL), although the difference was not significant $(p=0.624)$. However, the SRTs in a quiet environment with PSAP were reduced to $34.1 \pm 5.9 \mathrm{~dB}$ HL, which was significantly different from the unaided $(p<$ $0.001)$ and HA-aided conditions $(p<0.001)$.

Fig. 3B shows the SRTs in a noisy condition. A repeated measures ANOVA determined that SNR thresholds differed significantly between listening conditions $[\mathrm{F}(2,36)=5.645, p=$ 0.007]. However, Bonferroni-corrected post hoc tests revealed that the HA and PSAP devices failed to reduce SNR 
thresholds compared to the unaided condition ( $p=0.940$ for HA-aided vs. unaided and $p=0.160$ for PSAP-aided vs. unaided conditions). The SNR threshold was lowest with PSAPs (mean SNR threshold of $-2.4 \mathrm{~dB} \mathrm{HL}$ ), followed by the unaided condition (mean SNR threshold of $-1.7 \mathrm{~dB}$ HL) and with HAs (mean SNR thresholds of $-1.3 \mathrm{~dB} \mathrm{HL}$ ).
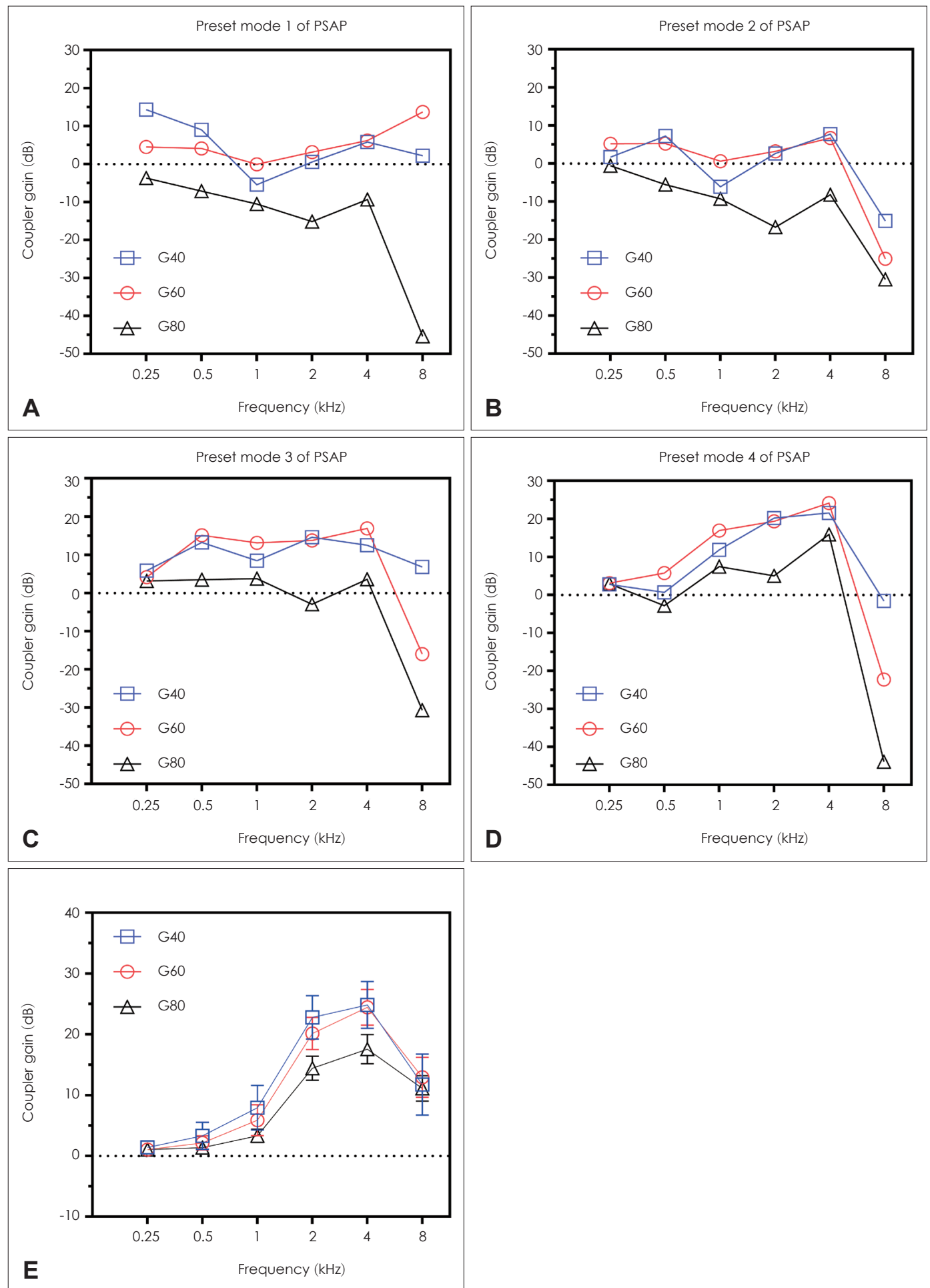

Fig. 1. Coupler gains of personal sound amplification product (PSAP) and digital hearing aid (DHA). Coupler gains were measured at inputs of 40,60, and $80 \mathrm{~dB}$ SPL (G40, G60, and G80). (A-D) Coupler gains of the PSAP according to four amplification preset mode. (E) Means with $95 \%$ confidence interval of the DHA's coupler gains after fitting with National Acoustic Laboratories' non-linear prescription, version 2. 

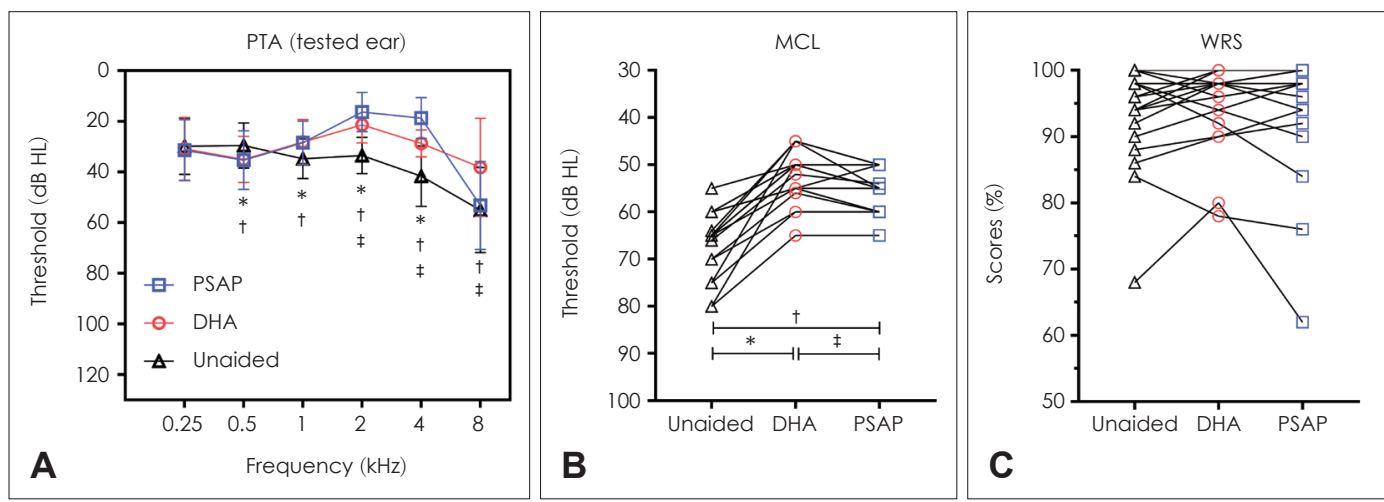

Fig. 2. Audiograms for the unaided, digital hearing aid (DHA)-aided, and personal sound amplification product (PSAP)-aided test conditions. Pure-tone thresholds (A) at each frequency (from $250 \mathrm{~Hz}$ to $8 \mathrm{kHz}$ ), most comfortable levels (MCL, B), and word recognition scores (WRS, C). The WRS was measured at MCL. Symbols indicate statistically significant differences in post hoc analysis ( ${ }^{*}$ for unaided vs. DHAaided conditions, tfor unaided vs. PSAP-aided conditions, ffor DHA-aided vs. PSAP-aided conditions). PTA: pure-tone audiometry.
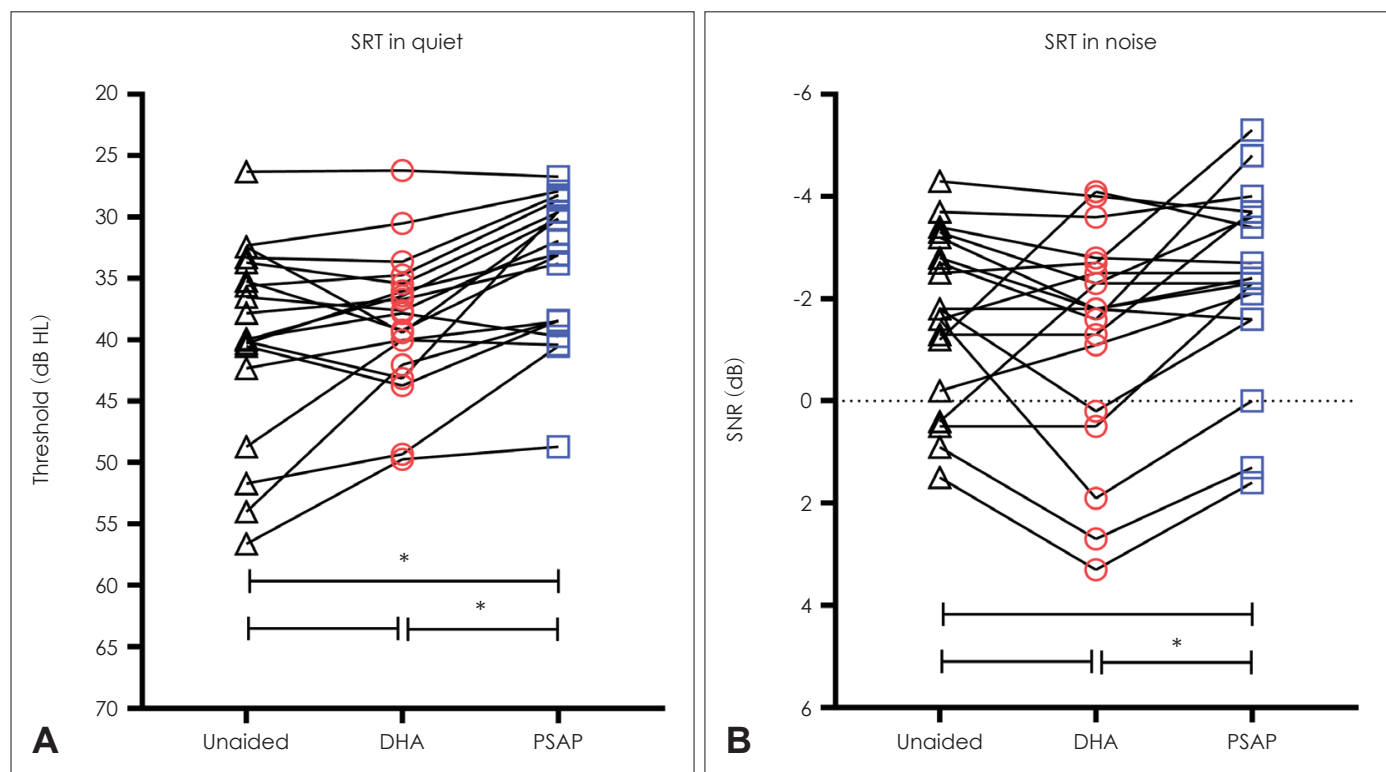

Fig. 3. Speech intelligibility under quiet (A) and noisy (B) test conditions. Speech intelligibility for the unaided digital hearing aid (DHA)-aided, and personal sound amplification product (PSAP)-aided test conditions. An asterisk $\left(^{*}\right)$ indicates a statistically significant difference in post hoc analysis. SRT: speech reception thresholds, SNR: speech to noise ratio.

\section{Speech quality and device preference}

Fig. 4A shows the MOS of speech quality among the tested listening conditions. A Friedman test identified no significant differences in speech quality in a quiet environment [ARC; $\chi^{2}(2)=1.000, p=0.607$ ], speech distortion [SIG; $\chi^{2}(2)=2.711$, $p=0.258]$, and noise intrusiveness $\left[\mathrm{BAK} ; \chi^{2}(2)=0.897, p=\right.$ 0.639 ] depending on listening conditions. There was a significant difference in speech quality in a noisy environment depending on listening conditions [AVR; $\left.\chi^{2}(2)=7.849, p=0.020\right]$. However, post hoc analysis with Wilcoxon signed-rank tests (significance level set at $p<0.017$ with a Bonferroni correction) revealed no significant differences between the results reported by unaided and HA-aided participants $(\mathrm{Z}=-1.278$, $p=0.201$ ) or between the unaided and PSAP-aided partici- pants $(\mathrm{Z}=-1.104, p=0.269)$. The highest MOS was reported by PSAP-aided participants (mean of 3.4), followed by unaided (mean of 3.0) and HA-aided participants (mean of 2.7). There was no statistically significant difference in speech quality as reported by PSAP- or HA-aided particiapnts when in the noisy testing environment $(\mathrm{Z}=-2.229, p=0.026)$.

Fig. 4B shows the participants' subjective device preferences when tested in either the quiet or noisy environment. In the quiet environment, eight participants preferred an $\mathrm{HA}$ and eight participants preferred a PSAP. The remaining three participants had no preference in hearing device. The mean CMOS was -0.5 , and in the quiet environment the participants preferred the PSAP to the HA. When tested in the noisy environment, eight participants preferred the HA and nine partici- 

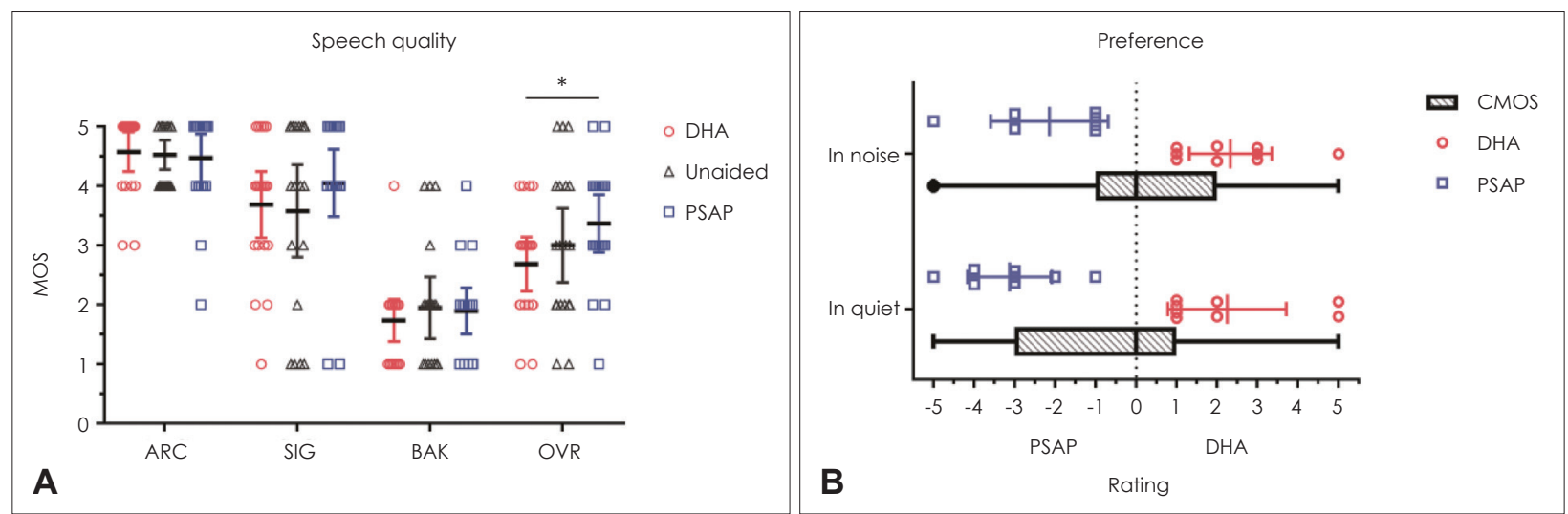

Fig. 4. Mean opinion scores (MOS) of speech quality and comparison mean opinion scores (CMOS) of device preference with $95 \%$ confidence intervals. The absolute category rating of speech quality (ARC) and speech signal distortion (SIG) in a quiet background, noise intrusiveness (BAK), and speech quality in a noisy background (OVR) are shown for the unaided, digital hearing aid (DHA), and personal sound amplification product (PSAP) conditions (A). The comparison mean opinion score for device preference in a quiet and noisy background are shown as box-and-whisker plots, except for a reading of 0 , which indicates no device preference (B). Bars and error bars represent means and $95 \%$ confidence intervals. An asterisk $\left({ }^{*}\right)$ indicates a statistically significant difference in Friedman test.

pants preferred the PSAP, and the mean CMOSs was 0.2.

\section{Discussion}

This study directly compared speech performance, sound quality, and preference between a PSAP and a basic HA among individuals with mild-to-moderate SNHL. Speech intelligibility in a quiet environment was significantly improved after wearing a PSAP, and this improvement was better than the result obtained with an HA. The PSAP also demonstrated significant improvement in the MCL in a quiet environment, similar to the MCL with an HA. However, speech intelligibility in a noisy environment was slightly improved after wearing a PSAP compared to the unaided condition (change from unaided, $0.7 \mathrm{~dB}$; $95 \% \mathrm{CI},-1.1$ to 0.4 ), which was not significant. Previously, Reed, et al. [15] reported that select PSAPs improved speech understanding in a noisy environment for those with mild-to-moderate hearing loss, but our results were insufficient to demonstrate the benefit of wearing a PSAP for improvement of speech intelligibility in a noisy environment. Subjective ratings of overall speech quality in the noisy testing condition also revealed that the PSAP elicited a slightly higher score compared to the unaided condition, but this was not significant.

Although the PSAP could not provide improvement in puretone thresholds at $8 \mathrm{kHz}$, it significantly improved hearing thresholds at 1, 2, and $4 \mathrm{kHz}$. The DHA fitted with a NALNL2 provided more functional gain at high frequency $(8 \mathrm{kHz})$, but less functional gain at mid frequencies ( 2 and $4 \mathrm{kHz}$ ) than the PSAP in pure-tone audiometry. Generally, NAL-NL2 prescribes less overall gain and a higher compression ratio than NAL-NL1 for adults with mild or moderate hearing loss
$[20,25]$. Also, NAL-NL2 prescribes relatively more gain across low and high frequencies and less gain across mid frequencies than NAL-NL1. Because the Korean language involves more energy across low frequencies and less energy across high frequencies than English, according to a longterm average speech spectrum comparison [26], the more functional gain at 2 and $4 \mathrm{kHz}$ might contribute to better speech intelligibility in a quiet environment with a PSAP than an HA for Koreans with mild-to-moderate hearing loss. In this study, most participants ( 10 of 19) selected the PSAP mode with the smallest amount of amplification. These results suggest that the slight amplification provided by a PSAP could provide sufficient improvement in speech intelligibility in a quiet environment for individuals with mild-to-moderate hearing loss. However, this slight amplification was insufficient to improve speech intelligibility in a noisy environment. In addition, the PSAP did not provide a functional gain at $8 \mathrm{kHz}$, and this gain frequency response indicates that the PSAP was not suitable for high frequency hearing loss.

This study has several limitations. First, this study is limited by a lack of acclimatization and verification to maximize the benefit of HAs. Use of an HA rather degraded speech intelligibility in a noisy environment in 11 participants. Subjective ratings on speech quality in a noisy background also revealed that the HA elicited the lowest score among listening conditions ( $2.7 \pm 0.9$ for DHA, $3.0 \pm 1.3$ for unaided, and $3.4 \pm 1.0$ for PSAP, OVR). The HA might produce speech distortion or background noise intrusiveness for new users, causing greater impairment of intelligibility when noise is mixed with speech [26]. Second, since only one PSAP and HA were used in the study, our results are limited to generalizations. Further research using a wider range of PSAPs and HAs is 
needed in order to better generalize the abilities of PSAPs relative to HAs. Third, the results of long-term PSAP and HA use in real life were not analyzed. Finally, all functions of the HA were disabled other than noise reduction, automatic directionality, and feedback cancellation.

Although our study has several limitations, the PSAP showed similar or better speech intelligibility in a quiet environment compared to an HA in individuals with mild-to-moderate SNHL. There was no significant subjective preference between the PSAP and HA. However, our results were not sufficient to demonstrate the benefit of PSAP on speech intelligibility in a noisy background. Therefore, this study suggests that PSAPs provide considerable improvement of speech intelligibility in a quiet environment and can be a good alternative to compensate for mild-to-moderate hearing loss; however, further research is required to verify these results.

\section{Conflicts of interest}

The authors have no financial conflicts of interest.

\section{Author Contributions}

Conceptualization: Sung Hwa Hong and Il Joon Moon. Data curation: Jinryoul Kim. Formal analysis: Ji Eun Choi. Methodology: Jinryoul Kim and Sung Hoon Yoon. Project administration: Sung Hwa Hong and Il Joon Moon. Resources: Sung Hwa Hong. and Il Joon Moon. Software: Jinryoul Kim and Sung Hoon Yoon. Supervision: Il Joon Moon. Validation: Ji Eun Choi Visualization: Ji Eun Choi. Writing — original drift: Ji Eun Choi. Writing—review \& editing: Ji Eun Choi and Il Joon Moon.

\section{ORCID iDs}

\section{Ji Eun Choi}

Jinryoul Kim

Sung Hoon Yoon

Sung Hwa Hong

Il Joon Moon
https://orcid.org/0000-0001-8105-813X https://orcid.org/0000-0002-9260-6370 https://orcid.org/0000-0002-6593-9997 https://orcid.org/0000-0002-2140-5141 https://orcid.org/0000-0002-3613-0734

\section{REFERENCES}

1) Mick P, Kawachi I, Lin FR. The association between hearing loss and social isolation in older adults. Otolaryngol Head Neck Surg 2014;150:378-84.

2) Dalton DS, Cruickshanks KJ, Klein BE, Klein R, Wiley TL, Nondahl DM. The impact of hearing loss on quality of life in older adults. Gerontologist 2003;43:661-8.

3) Monzani D, Galeazzi GM, Genovese E, Marrara A, Martini A. Psychological profile and social behaviour of working adults with mild or moderate hearing loss. Acta Otorhinolaryngol Ital 2008;28:61-6.

4) Scherer MJ, Frisina DR. Characteristics associated with marginal hearing loss and subjective well-being among a sample of older adults. J Rehabil Res Dev 1998;35:420-6.

5) Moon IJ, Baek SY, Cho YS. Hearing aid use and associated factors in South Korea. Medicine (Baltimore) 2015;94:e1580.

6) Abrams HB, Kihm J. An introduction to MarkeTrak IX: a new baseline for the hearing aid market. Hearing Review 2015;22:16.

7) Choi JE, Ahn J, Park HW, Baek SY, Kim S, Moon IJ. Prevalence of minimal hearing loss in South Korea. PLoS One 2017;12:e0171635.

8) Kochkin S. MarkeTrak VIII: the key influencing factors in hearing aid purchase intent. Hearing Review 2012;19:12-25.

9) Kochkin S. MarkeTrak VII: obstacles to adult non-user adoption of hearing aids. Hear J 2007;60:24-51.

10) Manchaiah V, Taylor B, Dockens AL, Tran NR, Lane K, Castle M, et al. Applications of direct-to-consumer hearing devices for adults with hearing loss: a review. Clin Interv Aging 2017;12:859-71.

11) Brody L, Wu YH, Stangl E. A comparison of personal sound amplification products and hearing aids in ecologically relevant test environments. Am J Audiol 2018;27:581-93.

12) Kochkin S. MarkeTrak VIII: utilization of PSAPs and direct-mail hearing aids by people with hearing impairment. Hearing Review 2010;17:12-6.

13) Smith C, Wilber LA, Cavitt K. PSAPs vs hearing aids: an electroacoustic analysis of performance and fitting capabilities. Hearing Review 2016;23:18.

14) Reed NS, Betz J, Lin FR, Mamo SK. Pilot electroacoustic analyses of a sample of direct-to-consumer amplification products. Otol Neurotol 2017;38:804-8.

15) Reed NS, Betz J, Kendig N, Korczak M, Lin FR. Personal sound amplification products vs a conventional hearing aid for speech understanding in noise. JAMA 2017;318:89-90.

16) Cheng CM, McPherson B. Over-the-counter hearing aids: electroacoustic characteristics and possible target client groups. Audiology 2000;39:110-6.

17) Jang H, Lee J, Lim D, Lee K, Jeon A, Jung E. Development of Korean standard sentence lists for sentence recognition tests. Audiol Speech Res 2008;4:161-77.

18) Johnson EE, Dillon H. A comparison of gain for adults from generic hearing aid prescriptive methods: impacts on predicted loudness, frequency bandwidth, and speech intelligibility. J Am Acad Audiol 2011;22:441-59.

19) Keidser G, Dillon H, Flax M, Ching T, Brewer S. The NAL-NL2 prescription procedure. Audiol Res 2011;1:e24.

20) Carhart R, Jerger JF. Preferred method for clinical determination of pure-tone thresholds. J Speech Hear Disord 1959;24:330-45.

21) Moon SK, Kim SH, Mun HA, Jung HK, Lee JH, Choung YH, et al. The Korean hearing in noise test. Int J Audiol 2008;47:375-6.

22) Nilsson M, Soli SD, Sullivan JA. Development of the hearing in noise test for the measurement of speech reception thresholds in quiet and in noise. J Acoust Soc Am 1994;95:1085-99.

23) International Telecommunication Union (ITU). P.800: Methods for subjective determination of transmission quality [Internet]. ITU; 1996 [cited 2015 May 8]. Available from: URL: https://www.itu. int/rec/T-REC-P.800-199608-I.

International Telecommunication Union (ITU). P. 835. Subjective test methodology for evaluating speech communication systems that include noise suppression algorithm [Internet]. ITU; 2003 [cited 2015 May 8]. Available from: URL: https://www.itu.int/rec/T-RECP.835/en.

24) Park G, Lee JH, Kim KS. Study on effective improvement of mobile phone sound quality in a noise environment for the hearing-impaired. Korean J Audiol 2013;17:78-82.

25) Licklider JCR. Effects of amplitude distortion upon the intelligibility of speech. J Acoust Soc Am 1946;18:429-34. 